Article

\title{
Advanced Multi-Color Fluorescence Imaging System for Detection of Biotic and Abiotic Stresses in Leaves
}

\author{
Stefanie Konanz ${ }^{1}$, László Kocsányi ${ }^{2}$ and Claus Buschmann ${ }^{1, *}$
}

1 Botanical Institute II, Karlsruhe Institute of Technology (KIT), University Sector, Fritz-Haber-Weg 4, 76131 Karlsruhe, Germany; E-Mail: stefanie.konanz@kit.edu

2 Department of Atomic Physics, Budapest University of Technology and Economics, Budafoki út 8, 1111 Budapest XI, Hungary; E-Mail: kocsanyi@eik.bme.hu

* Author to whom correspondence should be addressed; E-Mail: claus.buschmann@kit.edu; Tel.: +49-721-6084-4876; Fax: +49-721-6084-4874.

Received: 30 January 2014; in revised form: 20 March 2014 / Accepted: 25 March 2014 /

Published: 4 April 2014

\begin{abstract}
The autofluorescence of a sample is a highly sensitive and selective optical property and gives the possibility to establish non-destructive techniques of the investigation of plants, like detecting the chlorophyll fluorescence related to stress phenomena. In this study, an advanced multi-color fluorescence imaging system and data analysis were presented. The advantage of an imaging system is the additional receiving of spatial information over a sample area, this is a strong improvement compared to spot measurements commonly used. The purpose was to demonstrate the possibility of the detection and characterization of stress symptoms using this system. Specific fluorescence ratios were identified to characterize the stress status over the whole leaf, here shown on barley grown under different nitrogen supply (abiotic stress). Due to the changes, it is possible to make conclusions about leaf pigments (chlorophylls and phenolics) related to stress response. The second aim was to use the shape of local symptoms (biotic stress) as a criterion. For this purpose, three structural different kinds of fungal symptoms were analyzed using shape descriptors. It shows that an additional image shape analysis can be very useful for extracting further information, in this case the successful discrimination of fungal infections.
\end{abstract}

Keywords: fluorescence image; stress detection; symptom characterization; shape analysis; ImageJ; leaf level 


\section{Introduction}

The measurement of fluorescence gives the possibility for highly accurate and sensitive non-destructive detection method of specific fluorescence dyes (for a general description see e.g., [1]). Fluorescence represents a part of the energy taken up by light absorption (excitation) of the fluorescence dye. The fluorescence dyes, which cause the autofluorescence of plant material, are chlorophyll $a$ and secondary plant products like cell wall bound ferulic acid [2-4]. Furthermore, stress-induced phenolic compounds can also be detected with this method, expressed as additional blue-green fluorescence signature [5-7].

Another issue, the fluorescence signal of a plant tissue is strongly influenced by the localization of fluorescence dyes within the sample and the optical properties of cellular tissue structure. In the case of plant tissue, a part of the incident light can be hindered on its way to the fluorescence dye. For example, UV-absorbing substances in the epidermis of a leaf are shielding the chlorophyll $a$, which is located nearly exclusively in the cell layers below the epidermis [8]. Furthermore, the fluorescence emission is often changed on its path to the sample surface where it can be detected. One example is the re-absorption of chlorophyll fluorescence caused by the overlapping of the fluorescence emission spectrum with the absorption spectrum of the leaf pigments located on the way of fluorescence signal to the leaf surface [9]. The red and far-red chlorophyll fluorescence of intact leaves with photosynthetic activity is characterized additionally by two unique features [10]: (a) the absorption of light by accessory pigments (carotenoids in higher plants) contributes to the excitation of chlorophyll fluorescence because an energy transfer to the chlorophylls takes place in the antenna systems and (b) the yield of the chlorophyll fluorescence is also determined by photosynthetic activity. This is usually resulting in an inverse relationship between chlorophyll fluorescence and photosynthetic activity.

In summary, the fluorescence emitted from plant material is a complex signal and can be influenced by the penetration of the excitation light, the re-absorption of the fluorescence on its way to the sample surface, and - in case of chlorophyll fluorescence-energy transfer processes and photosynthetic activity. This gives room for specific detection of sample characteristics and changes of plant material caused by diseases or abiotic stresses.

Fluorescence images of plant samples have been shown useful to characterize the heterogeneity and changes within the measuring area [11]. It is also a way to get a higher statistical confidence than with averaged single spot measurements (see [12-15] for reviews). Additionally, the combination of different excitation lights and emission bands make it possible to measure specific fluorescence signals relevant for the detection and characterization of various stress factors of plants [12,14].

In the past, our research group set up a first version of the Fluorescence Imaging System (FIS), which was tested at several tasks in basic and applied research (described in detail [16]). Here, we present the advanced second generation of a multi-color FIS for detecting plant diseases and abiotic stress factors. This system, with new, state-of-the-art components (outstanding high filter transmittance and extremely precise synchronization setting), is highly flexible and allows a rapid and non-destructive monitoring of plant material and informs about the health status. A detailed image analysis with the selection of relevant regions of interest (fungal infection) was demonstrated, and the use of shape descriptors made it possible to discriminate different types of symptoms depending on the shape. 


\section{Results and Discussion}

\subsection{Fluorescence Image Analysis of Local Fungal Symptoms Using ImageJ}

In order to make use of the full spatial information of an image related to a biological issue, here for stress detection, the processing should be complemented by a detailed image analysis using the appropriate software application. In this study, the task was accomplished by using the open-source software ImageJ, which can effectively solve image analysis challenges, and it is widely used as biological imaging tool [17]. Figures 1 and 2 illustrate the general steps to recognize the leaf margin and identify fungal symptoms (biotic stress).

The first objective is to separate the leaf from the background. The automated threshold-function was applied to one image showing a clear discrimination between background and leaf, in our case the

${ }^{447}$ F689 band (superscript number: wavelength of excitation; number behind F: wavelength of fluorescence). The obtained ROI (region of interest) is equal to the leaf area and can be saved and applied to all other fluorescence bands. Afterwards, the clear outside-command set all pixels outside the leaf to zero (black color). Furthermore, the threshold-function can also be used for the identification of the symptoms (ROIs). At the beginning, it can be necessary to manually adjust the threshold values for the different fluorescence bands. Once the function has been established and all symptoms are identified and saved as ROIs the measure-command can be operated, a list of possible parameters (e.g., area or shape descriptors) is available. If there are multiple ROIs, a useful tool is the ROI-manager, which allows it to analyze a data set in one operation step. Figure 1 demonstrates the procedure for a sugar beet leaf naturally infected with leaf spots (Cercospera beticola). The fluorescence image was excited with blue light and shows the red chlorophyll $a$ fluorescence $\left({ }^{447} \mathrm{~F} 689\right.$; Figure 1A,C). As a result, the measure-function calculates $0.81 \%$ infected area and 27 single spots (Figure 1C).

The second example presents the fluorescence images of a grapevine leaf infected with black rot pathogen (Guignardia bidwellii) 11 days after inoculation (11 dai). Here, two representative fluorescence bands were chosen: the UV-A-excited blue fluorescence $\left({ }^{340} \mathrm{~F} 440\right)$ and blue-excited red fluorescence $\left({ }^{447} \mathrm{~F} 689\right)$ images. The comparison of both images (Figure 2A,B) shows clearly a larger elongation of the fungal symptoms in the ${ }^{340} \mathrm{~F} 440$ fluorescence band. The combined illustration in Figure $2 \mathrm{C}$, and the analysis of the ROIs confirms this impression. The measure-command detects a 2.1-fold larger infected area at the ${ }^{340} \mathrm{~F} 440$ fluorescence band.

This phenomenon was also observed in green fluorescence images excited with UV-A $\left({ }^{340} \mathrm{~F} 550\right)$, but not quite so pronounced (image not shown, [18]). The larger elongation of the F440 fluorescence symptoms can be explained by a change in the chemical composition of blue fluorescing phenolic compounds at the infection site. The stress-induced response, accumulation of phenolics, to the fungal attack can be monitored in this case [5,19]. Furthermore, the smaller visible symptoms (Figure 2D) correspond to all red and far-red fluorescence images independently of the excitation light (see Figure 2B as an example).

The fluorescence image processing and analyzing procedure using ImageJ was the same as described above. In addition, here, the threshold-tool was applied to set the lower and upper values of the function, segmenting the image into features of interest and background. 
Figure 1. Image analysis of sugar beet leaf spots (Cercospora beticola) caused by a natural infection in the field as an example of a general workflow using ImageJ. (A) Image of the blue-excited chlorophyll $a$ fluorescence at $689 \mathrm{~nm}\left({ }^{447} \mathrm{~F} 689\right)$. The larger circular spots are caused by black disc magnets using to flatten the sugar beet leaf (see also panel D). The leaf margin and the magnets are highlighted by a green line; (B) Application of the threshold-function (ImageJ) to the fluorescence image in panel A. The original fluorescence image was converted into a binary image automatically. The orange lines are equivalent to the outer border of the symptoms; (C) Visualization (light blue) and labeling of the individual ROIs (regions of interest) at the original fluorescence image $\left({ }^{447} \mathrm{~F} 689\right)$. The used ROI manager-function allows also the saving of the ROIs (disease symptoms) for the further analyzing process; (D) Photograph of the infected sugar beet leaf.
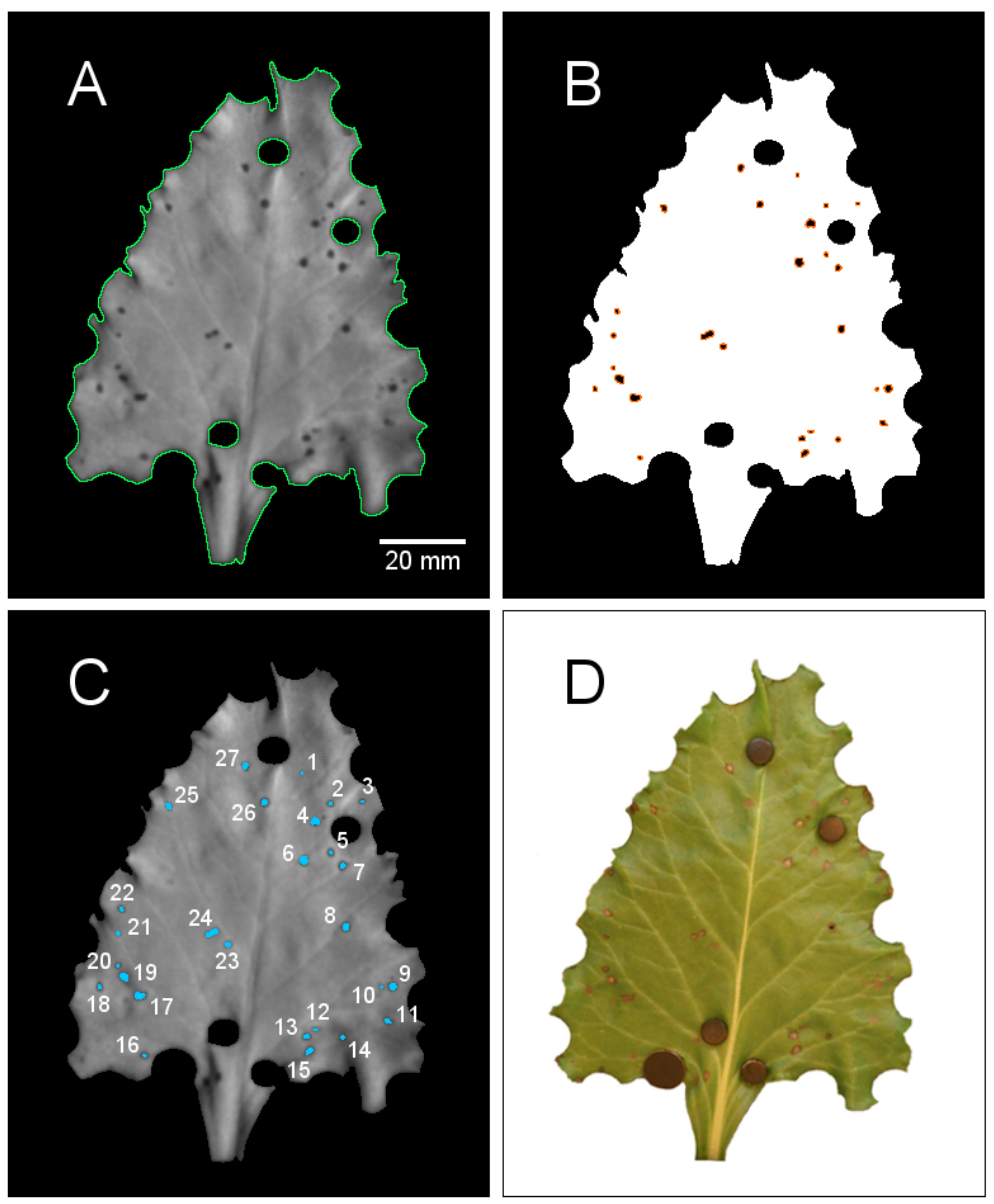

\subsection{Characterization of Fungal Symptoms Using Shape Analysis}

In this part, the two experiments above served as basis for an extended data analysis using the shape as criterion for symptom characterization. The sugar beet leaf spots (Figure 1) represent a small and more compact shape of fungal symptoms. Otherwise, the grapevine leaf infected with black rot (Figure 2) developed symptoms with more or less rough boundary and a larger elongation depending on the fluorescence band used. 
Figure 2. Images of a grapevine leaf infected with the black rot disease caused by Guignardia bidwellii 11 days after an artificial spray inoculation (11 dai (days after inoculation)). Two different fluorescence images are presented: (A) UV-A-excited blue fluorescence $\left({ }^{340} \mathrm{~F} 440\right)$ and (B) fluorescence at $689 \mathrm{~nm}$ excited with blue light $\left({ }^{447} \mathrm{~F} 689\right)$; (C) Joint presentation of the ROIs (regions of interest) obtained from the disease symptoms of the fluorescence images in panel A and B. The distribution of the symptoms is shown as colored areas, panel A and B are displayed in orange and light blue areas, respectively; (D) Photograph of the infected grapevine leaf.
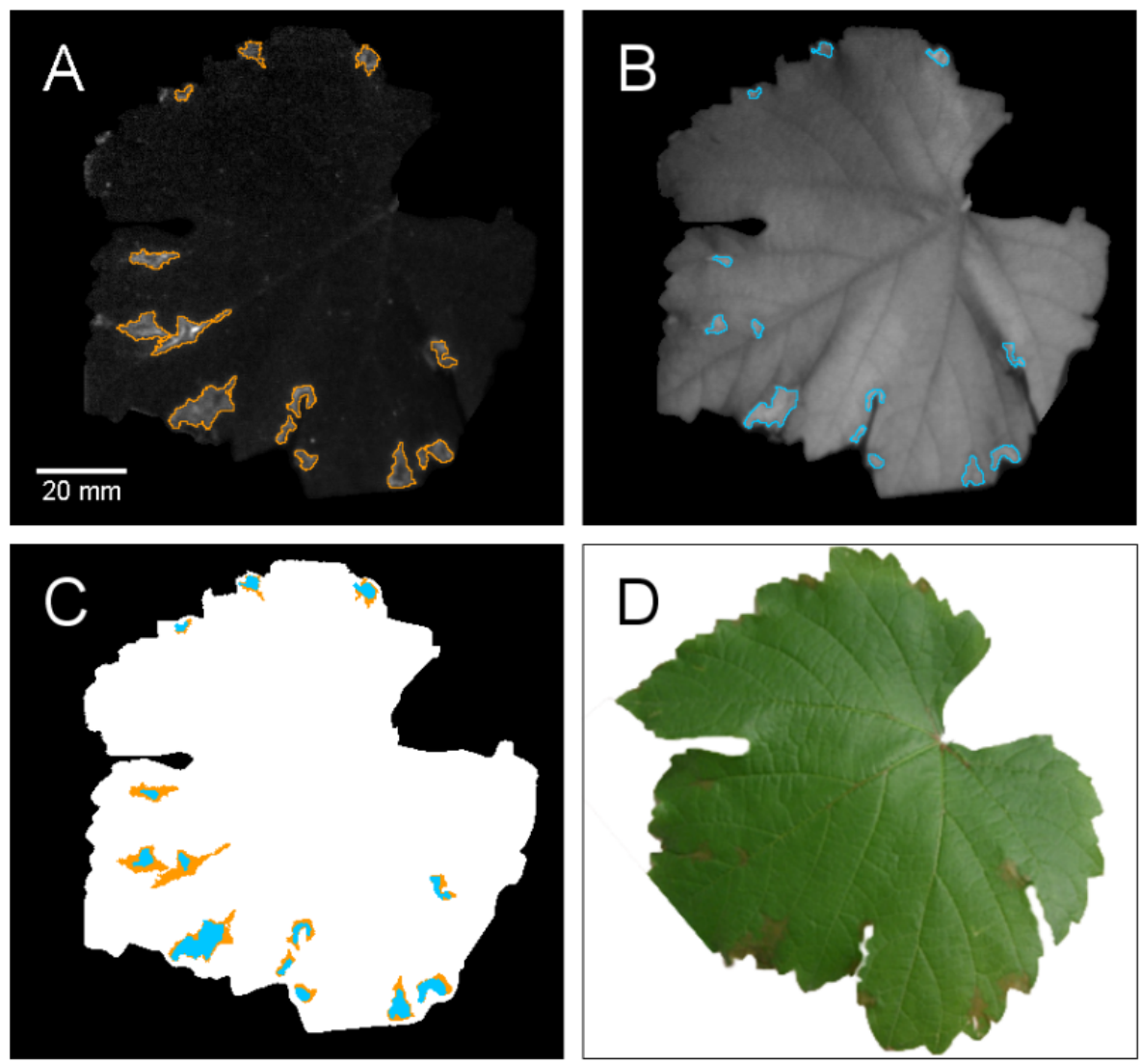

For this step, the ROIs of Figures $1 \mathrm{C}$ and $2 \mathrm{C}$ were used to qualitatively describe the shape of the symptoms to obtain additional information. The analysis of the shape descriptors is based on the previous defined thresholds and the resulting ROIs. The measure-command of the software ImageJ calculated a set of four shape parameters: circularity, solidity, roundness, and aspect ratio. The plots in Figure 3 represent the distribution of the four shape parameters as functions of the frequency of occurrence for the three different types of fungal symptoms. For reason of comparability, the frequency results were normalized to 100 .

Generally, in all four graphs the distribution of the shape descriptor related to sugar beet leaf spots are closer to the value 1 than for the black rot symptoms. The reason is the more compact and round shape of these symptoms. Related to the most irregular shape and largest elongation, the grape black rot symptoms at ${ }^{340} \mathrm{~F} 440$ are located always to the farthest left followed by the other black rot band $\left({ }^{447} \mathrm{~F} 689\right)$. 
Figure 3. Histograms of four shape parameters analyzed from the fungal symptoms, the data basis was the individual ROIs (fungal symptoms) shown in Figure 1C (sugar beet leaf spots) and Figure 2A, B (grape black rot). Four shape descriptors (circularity, solidity, roundness, aspect ratio) were calculated and the frequency distribution was displayed graphically as histograms. The orange and blue bars are representing the results of the grape black rot symptoms, based on the UV-A-excited blue $\left({ }^{340} \mathrm{~F} 440\right)$ and the blue-excited red fluorescence $\left({ }^{447} \mathrm{~F} 689\right)$, respectively. The analysis of the symptoms of sugar beet leaf spots $\left({ }^{447} \mathrm{~F} 689\right)$ is displayed as grey bars in the histograms.

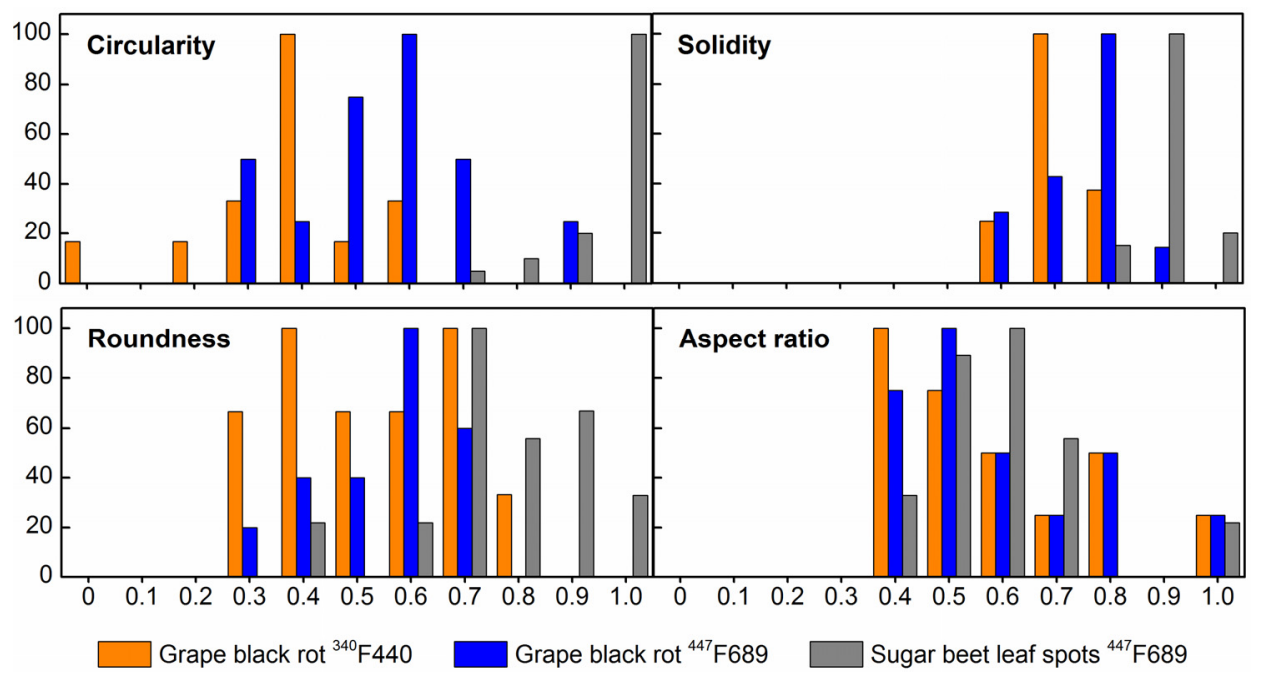

The aspect ratio shows a more or less overlapping of the three distributions and can therefore not be considered for our purpose. The parameter solidity is sensitive to the shape of an image region (here symptom), resulting in more or less density distributions, and all three fungal symptoms are close to each other. In comparison, the shape descriptor roundness, influenced by elongation, shows a wider distribution individually, but a better discrimination of the three types of symptoms than solidity.

It shows that circularity was the most appropriate parameter with the highest discrimination between the three different types of symptoms, especially for the two fluorescence bands caused by grape black rot disease. This shape descriptor is determined by overall elongation and boundary irregularity of an image region. This result indicates the more variable nature of this parameter, because it is the only one sensitive to two factors: elongation and shape.

\subsection{Fluorescence Image Analysis of Abiotic Stress Symptoms Extended over the Entire Leaf}

The reference measurements from barley leaves grown under different nitrogen (N) supply gave the following results. The photosynthetic active pigments were increasing constantly with a higher $\mathrm{N}$-fertilization (Table 1). In the end $100 \% \mathrm{~N}$ was 3.3 -fold higher for chlorophylls $(\mathrm{Chl} a+b)$ and 2.6-fold for carotenoids $(\operatorname{Car} x+c)$ in relation to the $\mathrm{N}$-deficient variant $(0 \% \mathrm{~N})$. The resulting rising ratio $(a+b) /(x+c)$ displays the lower accumulation of chlorophylls compared to carotenoids under stress conditions [20], here ranging from 4 to 5 (Table 1). This is in good agreement with reports on N-deficient barley [21], maize [22], and sugar beet [23]. The SPAD-values, another indicator of the Chl content, increased in the same range as the ratio $(a+b) /(x+c)$ with higher N-availability (1.9-fold), see Table 1. These results are similar to values in other N-deficiency studies [21,23-25]. 
Further, the measured barley plants with an optimal N-treatment $(100 \% \mathrm{~N})$ contained $64 \%$ and $32 \%$ more $\mathrm{N}$ than those of the $0 \%$ and $50 \% \mathrm{~N}$-supply, respectively. Optically, the leaves of 50\% and 100\% $\mathrm{N}$ looked healthy and dark-green, but the $\mathrm{N}$-deficiency supply $(0 \% \mathrm{~N})$ showed a reduced growth and green-yellow colored leaves with distinct chlorosis on the leaf tips (RGB images not shown, [26]).

Table 1. Different reference parameters were estimated at the last full developed leaf of barley plants (Hordeum vulgare L.) grown under low, middle and optimized nitrogen supply $(0 \%, 50 \%, 100 \% \mathrm{~N})$. The contents of chlorophyll $a(\mathrm{Chl} a)$ and $b(\mathrm{Chl} b)$ and total carotenoids (Car $x+c)$ in $\mathrm{mg}$ per $\mathrm{m}^{2}$ leaf area as well as the pigment ratio $(a+b) /(x+c)$ were detected in vitro. These leaf pigments $(n=6 \pm \mathrm{SD})$ and SPAD-values $(n=8 \pm \mathrm{SD})$ were determined using a $12 \mathrm{~mm}$ long part located in the middle of the leaves. The percentage of total nitrogen per dry mass was analyzed from an average of 10 plants of each nitrogen treatment. Statistical analysis of data was accomplished by one-way ANOVA followed by Scheffé test to analyze specific differences between means at the 1\% and 5\% level. All parameters at the three treatments are showing significant differences; expect the ratio $(a+b) /(x+c)$ at the level of $1 \%(p \leq 0.01)$.

\begin{tabular}{ccccccc}
\hline & $\begin{array}{c}\text { Chl } \boldsymbol{a} \\
{\left[\mathbf{m g} / \mathbf{m}^{2}\right]}\end{array}$ & $\begin{array}{c}\text { Chl } \boldsymbol{b} \\
{\left[\mathbf{m g} / \mathbf{m}^{2}\right]}\end{array}$ & $\begin{array}{c}\text { Car } \boldsymbol{x}+\boldsymbol{c} \\
{\left[\mathbf{m g} / \mathbf{m}^{2}\right]}\end{array}$ & $\begin{array}{c}\text { RATIO } \\
(\boldsymbol{a}+\boldsymbol{b}) /(\boldsymbol{x}+\boldsymbol{c})\end{array}$ & SPAD & $\begin{array}{c}\text { Total N per } \\
\text { Dry Mass \% }\end{array}$ \\
\hline $0 \% \mathrm{~N}$ & $156.6 \pm 33.3$ & $42.1 \pm 9.7$ & $49.4 \pm 9.4$ & $4.01 \pm 0.13$ & $29.7 \pm 3.6$ & 0.84 \\
$50 \% \mathrm{~N}$ & $341.4 \pm 47.5$ & $98.2 \pm 11.9$ & $89.6 \pm 13.4$ & $4.92 \pm 0.12$ & $46.4 \pm 3.0$ & 1.58 \\
$100 \% \mathrm{~N}$ & $502.9 \pm 55.5$ & $152.6 \pm 16.5$ & $128.7 \pm 15.7$ & $5.09 \pm 0.06$ & $55.7 \pm 4.0$ & 2.34 \\
\hline
\end{tabular}

The differences in N-supply can also be seen in the changes of fluorescence pattern of blue, red, and far-red fluorescence and the resulting ratios. Three false-color fluorescence ratio images of barley leaves are presented in Figure 4, for each treatment $(0 \%, 50 \%$, and $100 \% \mathrm{~N})$ one representative leaf was chosen.

The fluorescence ratio F689 to F740 excited with blue light (Figure 4 right and Table 2) shows a significantly difference between $0 \% \mathrm{~N}$-supply and the other treatments, but not between $50 \%$ and $100 \% \mathrm{~N}$. The same tendency in the results was obtained using UV-A and green light for the excitation (Table 2). Green light has the advantage to give information deeper from the leaf tissue than blue light, this was demonstrated in leaf cross-sections by detecting the Chl fluorescence using different kind of excitation light [27-30]. Generally, the red and far-red fluorescence with maxima around 690 and $740 \mathrm{~nm}$ is emitted by Chl $a$ of the photosystem II located in the mesophyll cells of leaves [31-33]. The resulting Chl emission ratio red to far-red fluorescence (F690/F740) is inversely correlated with the in situ Chl content of leaves, as shown before in various plants [9,34-37]. This ratio is sensitive to the Chl content because of the partial re-absorption of the emitted red Chl fluorescence (F690) by the absorption band of the leaf chlorophyll around $680 \mathrm{~nm}$. F740, in turn, is much less affected by re-absorption and serves as an internal reference $[33,38]$. This ratio has been used widely in numerous studies, e.g., Buschmann 2007 [9]. 
Figure 4. Fluorescence images of barley leaves (Hordeum vulgare L.) grown under different nitrogen supply expressed as ratios of two different fluorescence bands (see Table 2 for the values). Each ratio (panel) shows the same representative leaf at the level of $0 \%, 50 \%$, and $100 \%$ nitrogen (left to the right). The fluorescence ratio images are shown in false colors.
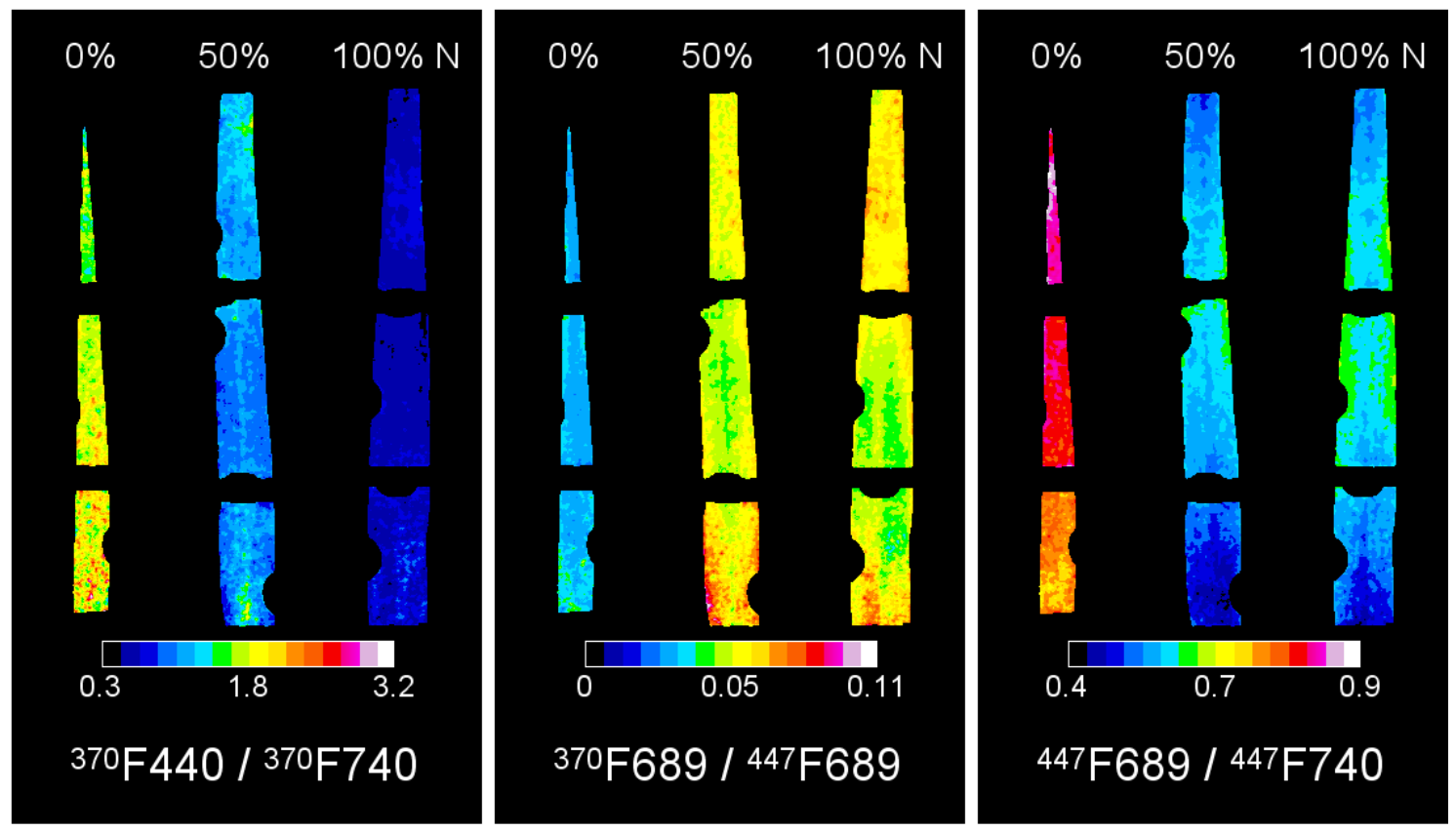

Table 2. Fluorescence ratios of barley leaves (Hordeum vulgare L.) grown under $0 \%, 50 \%$, and $100 \%$ nitrogen supply $(\mathrm{N})$. The underlying images were excited with UV-A $(370 \mathrm{~nm})$, blue $(447 \mathrm{~nm})$ or green light $(550 \mathrm{~nm})$, and detected at three bands (440, 689, and $740 \mathrm{~nm})$. The average fluorescence values were calculated from the middle part of the images (integrated values of all the pixels). The values ( \pm SD) are the result of eight barley leaves for each N-treatment. Mean values in the same column with different letters are significantly different (one-way ANOVA, Scheffé test)

\begin{tabular}{|c|c|c|c|c|c|c|c|c|c|}
\hline & $\frac{{ }^{370} \mathrm{~F} 689}{{ }^{370} \mathrm{~F} 740}$ & $p \leq 0.05$ & $p \leq 0.01$ & $\frac{{ }^{447} \mathrm{~F} 689}{{ }^{447} \mathrm{~F} 740}$ & $p \leq 0.05$ & $p \leq 0.01$ & $\frac{{ }^{550} \mathrm{~F} 689}{{ }^{550} \mathrm{~F} 740}$ & $p \leq 0.05$ & $p \leq 0.01$ \\
\hline $0 \% \mathrm{~N}$ & $0.66 \pm 0.04$ & $\mathrm{a}$ & $\mathrm{a}$ & $0.743 \pm 0.056$ & $\mathrm{a}$ & $\mathrm{a}$ & $0.511 \pm 0.050$ & $\mathrm{a}$ & $\mathrm{a}$ \\
\hline $50 \% \mathrm{~N}$ & $0.47 \pm 0.03$ & $\mathrm{~b}$ & $\mathrm{~b}$ & $0.551 \pm 0.060$ & $\mathrm{~b}$ & $\mathrm{~b}$ & $0.32 \pm 0.038$ & $\mathrm{~b}$ & $\mathrm{~b}$ \\
\hline \multirow[t]{2}{*}{$100 \% \mathrm{~N}$} & $0.44 \pm 0.03$ & $\mathrm{bc}$ & $\mathrm{bc}$ & $0.562 \pm 0.057$ & $\mathrm{bc}$ & $\mathrm{bc}$ & $0.28 \pm 0.029$ & $\mathrm{bc}$ & $\mathrm{bc}$ \\
\hline & $\frac{{ }^{370} \mathrm{~F} 440}{{ }^{370} \mathrm{~F} 689}$ & $p \leq \mathbf{0 . 0 5}$ & $p \leq 0.01$ & $\frac{{ }^{370} \mathrm{~F} 440}{{ }^{370} \mathrm{~F} 740}$ & $p \leq \mathbf{0 . 0 5}$ & $p \leq 0.01$ & $\frac{{ }^{370} \mathrm{~F} 689}{{ }^{447} \mathrm{~F} 689}$ & $p \leq 0.05$ & $p \leq 0.01$ \\
\hline $0 \% \mathrm{~N}$ & $2.19 \pm 0.37$ & $\mathrm{a}$ & $\mathrm{a}$ & $1.44 \pm 0.27$ & $\mathrm{a}$ & $\mathrm{a}$ & $0.032 \pm 0.004$ & $\mathrm{a}$ & $\mathrm{a}$ \\
\hline $50 \% \mathrm{~N}$ & $2.28 \pm 0.55$ & $\mathrm{a}$ & $\mathrm{a}$ & $1.08 \pm 0.28$ & $\mathrm{~b}$ & $\mathrm{a}$ & $0.047 \pm 0.006$ & $\mathrm{a}$ & $\mathrm{a}$ \\
\hline $100 \% \mathrm{~N}$ & $1.36 \pm 0.39$ & $\mathrm{~b}$ & $\mathrm{~b}$ & $0.6 \pm 0.18$ & $\mathrm{c}$ & $\mathrm{b}$ & $0.067 \pm 0.018$ & $\mathrm{~b}$ & $\mathrm{~b}$ \\
\hline
\end{tabular}

The use of an UV excitation source (multi-color system) gives the advantage of additional information, fluorescence bands in the region of 400 to $550 \mathrm{~nm}$. This naturally occurring blue-green fluorescence derives from hydroxycinnamic acids, mainly ferulic acid, covalently bound to cell walls of the leaf epidermis [4]. The change of the blue fluorescence signal can be associated with abiotic and 
biotic stresses in plants caused by phenolic compounds [12]. In this study, the relating fluorescence ratio ${ }^{370} \mathrm{~F} 440$ to ${ }^{370} \mathrm{~F} 740$ (Figure 4 left and Table 2) shows clearly a decreasing ratio for higher $\mathrm{N}$-supply and the means of all three treatments were significantly different from each other at the 0.05 level (Table 2). Both fluorescence bands are increasing with higher $\mathrm{N}$-amount, see Table 3 . The lower blue fluorescence in the N-deficient leaves occurs from higher amount of phenolic compounds with UV-absorbing properties related to the stress response in plants [6,7]. These phenolics are also involved in the epidermal UV protection [8] even against UV-A light [39].

Table 3. Fluorescence values of barley leaves (Hordeum vulgare L.) grown under $0 \%$, $50 \%$, and 100\% nitrogen supply $(\mathrm{N})$. The samples were excited with UV-A (370 nm), blue $(447 \mathrm{~nm})$ or green light $(550 \mathrm{~nm})$, and detected at three bands $(440,689$ and $740 \mathrm{~nm})$. The average fluorescence values were calculated from the middle part of the images (integrated values of all the pixels). The values $( \pm \mathrm{SD})$ are the result of eight barley leaves for each $\mathrm{N}$-treatment.

\begin{tabular}{|c|c|c|c|c|}
\hline & ${ }^{447} \mathrm{~F} 689$ & ${ }^{447} \mathbf{F} 740$ & ${ }^{550} \mathrm{~F} 689$ & ${ }^{550} \mathrm{~F} 740$ \\
\hline $0 \% \mathrm{~N}$ & $7036 \pm 579$ & $9469 \pm 443$ & $3981 \pm 205$ & $7836 \pm 725$ \\
\hline $50 \% \mathrm{~N}$ & $6330 \pm 1033$ & $11455 \pm 1036$ & $3406 \pm 390$ & $10662 \pm 765$ \\
\hline \multirow[t]{2}{*}{$100 \% \mathrm{~N}$} & $7261 \pm 1334$ & $12838 \pm 1311$ & $3473 \pm 499$ & $12356 \pm 664$ \\
\hline & ${ }^{370} \mathrm{~F} 440$ & ${ }^{370} \mathrm{~F} 689$ & ${ }^{370}$ F740 & \\
\hline $0 \% \mathrm{~N}$ & $495 \pm 93.3$ & $229 \pm 37.4$ & $346 \pm 49.8$ & \\
\hline $50 \% \mathrm{~N}$ & $667 \pm 178$ & $293 \pm 46.0$ & $621 \pm 87.7$ & \\
\hline $100 \% \mathrm{~N}$ & $620 \pm 120$ & $483 \pm 142$ & $1086 \pm 309$ & \\
\hline
\end{tabular}

For the same reason, the fluorescence band F740 excited with UV-A is increasing with higher $\mathrm{N}$-supply (Table 3). In the stressed $0 \% \mathrm{~N}$-variant the excitation light was absorbed to a larger degree by phenolics and consequently emitted less far-red fluorescence. In addition, the ratio ${ }^{370} \mathrm{~F} 440$ to ${ }^{370} \mathrm{~F} 689$ is influenced by a higher re-absorption of the red fluorescence band at increasing $\mathrm{N}$-availability (Table 2), because of the higher content of chlorophylls [29]. This is the reason for the slower rising of the red fluorescence (F689) than the far-red fluorescence band (F740), see Table 3. Both ratios have been found to be valid indicators of different stresses in plants including N-deficiency (see $[12,40]$ for reviews).

The ratio ${ }^{370} \mathrm{~F} 689 /{ }^{447} \mathrm{~F} 689$ shows the difference between Chl $a$ fluorescence excited with UV-A and blue light. This ratio describes the status of the epidermal UV-transmission of a leaf and subsequently the shielding effect against UV exposure [8,41]. The UV-A excited red fluorescence band can be indirectly affected by the amount of UV-absorbing phenolic compounds, which are stress-induced [6,7]. The fluorescence image and the mean values (Figure 4 middle and Table 2) give no significantly differences between $50 \% \mathrm{~N}$ and optimal $\mathrm{N}$-supply. The highly stressed $0 \% \mathrm{~N}$ treatment shows a lower ratio caused by a higher amount of phenolics as reaction. This describes the status of the epidermal UV-transmission of a leaf and subsequently the shielding effect against UV exposure [8,41]. The red fluorescence band is indirectly affected by amount of UV-absorbing substances, which are also related to abiotic and biotic stresses [6,7]. 


\section{Experimental Section}

\subsection{Plants and Treatments}

Sugar beet leaf spots: Sugar beet plants (Beta vulgaris L.) were grown outside in the Botanical Garden of the Karlsruhe Institute of Technology (Karlsruhe, Germany). The used cultivar Berenika was provided by the company KWS Saat AG (Einbeck, Germany). The measurements were carried out with leaves naturally infected with Cercospora beticola. The developmental stage of the plants was shortly before harvest, and only young expanded leaves of the inner part of the leaf rosette were chosen.

Grapevine black rot disease: four-months-old grapevine cuttings (Vitis vinifera L.) of the susceptible cultivar Müller-Thurgau were used. The infection with the black rot disease caused by Guignardia bidwellii was carried out using an artificial spray inoculation of the whole plants with a spore concentration of $10^{4}$ per $\mathrm{mL}$. During the inoculation period, the plants were maintained in the climate chamber under a plastic cover for $24 \mathrm{~h}$, afterwards transferred to a greenhouse. Growth and inoculation of the grapevine plants were carried out at the JKI (Julius-Kühn-Institute) for Grapevine Breeding (Geilweilerhof, Siebeldingen, Germany). The measurement was performed 11 days after infection, simultaneously with the first visible symptoms.

Barley under different nitrogen supply: Different nitrogen status was investigated using spring barley (Hordeum vulgare L. cv. Barke). The plants were grown in $18 \mathrm{~L}$ plastic pots $(270 \mathrm{~mm}$ height, $320 \mathrm{~mm}$ diameter), resulting in 30 plants per pot. The pots were filled with local field soil and placed outside at the research facility LTZ (Landwirtschaftliches Technologie Zentrum) Augustenberg at Karlsruhe (Germany). The pots allow adjustment of the nitrogen amount artificially. Three nitrogen concentrations were chosen: $0 \% \mathrm{~N}$ (no nitrogen added to the soil), $50 \% \mathrm{~N}(1.5 \mathrm{~g} / \mathrm{pot}$ ), and 100\% $\mathrm{N}$ ( $3 \mathrm{~g} / \mathrm{pot}$ ) equal with optimum nitrogen supply (according to agricultural standard) to support plant growth. For fluorescence imaging and reference measurements, the full developed leaf below the flag leaf was used at the stage of ear emergence for all three treatments.

\subsection{Multi-Color Fluorescence Imaging System}

The Multi-color FIS described here consists of an excitation light source (xenon lamp), the detection unit (ICCD camera), and a synchronization module. The individual elements are mounted on a flexible rail and carrier system (X95, Newport Spectra-Physics GmbH, Darmstadt, Germany). A computer is controlling the measurement and carrying out the image analysis (Figure 5).

\subsubsection{Excitation}

Fluorescence is excited by pulses of a xenon flash lamp (FX-4400 with Lite Pack FYD-1200, Perkin Elmer Optoelectronics, Cambridge, UK) with an input energy of 0.6 Joule per flash, a repetition rate of $50 \mathrm{~Hz}$, and a pulse width of approx. $7 \mu \mathrm{s}$. The xenon lamp power supply and ignition unit is equipped with a PIC (Peripheral Interface Controller) controller. It has the function to observe the video signal of the CCD and to synchronize flashes into the integrating (measuring) time sector of it. In addition to these, the controller sends a reference signal about the exact ignition time to the pulse generator, which produces the shutter signal of the image intensifier of the ICCD camera. The unit was 
designed and manufactured at the Department of Atomic Physics of the Budapest University of Technology and Economics. The excitation light illuminates the sample in a circular area with a diameter of $c a .300 \mathrm{~mm}$. The distance between the center of the measurement area and the lamp is $0.55 \mathrm{~m}$, and an angle of $30^{\circ}$ to the normal. A filter wheel (AI-FW50-7S, Apogee Imaging System, Roseville, CA, USA) with 7 positions for $50 \mathrm{~mm}$ square filters is mounted in front of the xenon lamp (see Figure 5). In this setting the following single-band bandpass filters (Figure 6) are installed: (1) $340 \mathrm{~nm}$ UV-A filter (DUG11, Schott, Mainz, Germany) with a central wavelength (CWL) of $340 \mathrm{~nm}$ and a bandwidth (BW) of $70 \mathrm{~nm}$; and three filters (BrightLine HC, Semrock, Rochester, NY, USA); (2) $370 \mathrm{~nm}$ UV-A filter (CWL $370 \mathrm{~nm} / \mathrm{BW} 36 \mathrm{~nm}$ ); (3) blue filter (CWL $447 \mathrm{~nm} / \mathrm{BW} 60 \mathrm{~nm}$ ); and (4) green filter (CWL $550 \mathrm{~nm} / \mathrm{BW} 88 \mathrm{~nm})$.

Figure 5. Setup of the Multi-color Fluorescence Imaging System for the measurement of leaf samples. (Left) Technical scheme of the assembling of the single components; (Right) Photograph of the sensor system.

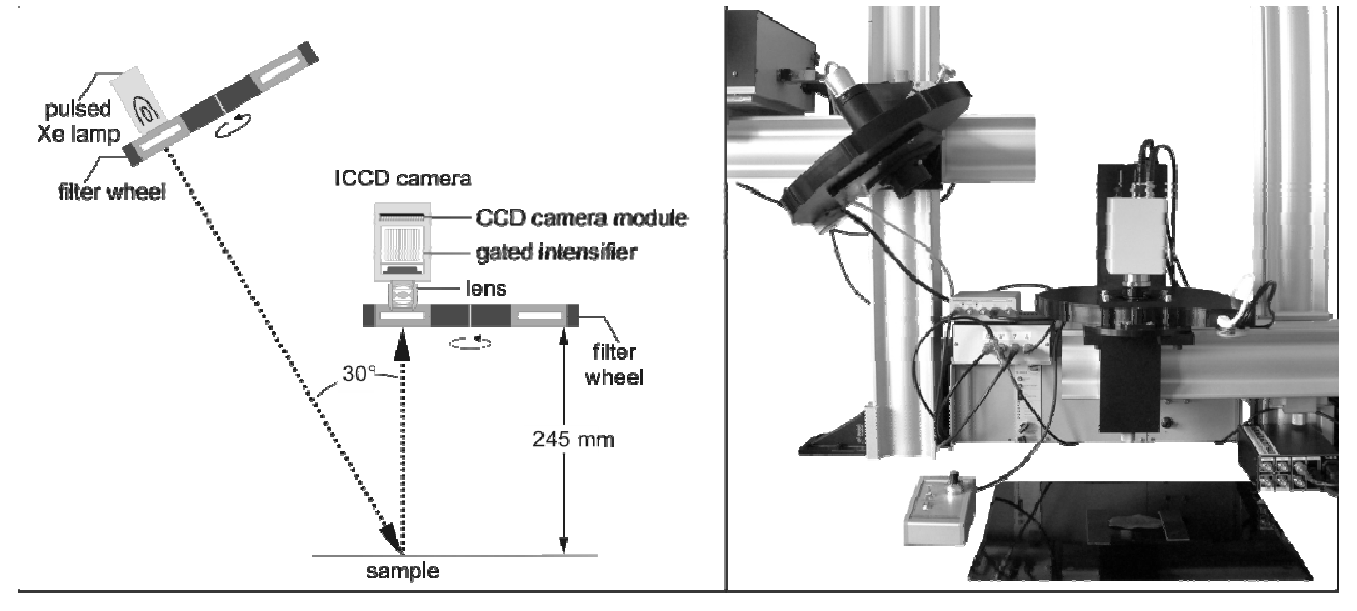

Figure 6. Transmittance spectra of the filters for excitation and detection in the two filter wheels. Excitation (Left): Two UV-A filters, a blue, and a green filter in front of the xenon lamp are producing different kind of light for fluorescence excitation; Detection (Right): Four filters for recording of blue, green, red, and far-red fluorescence bands are mounted in front of the camera.

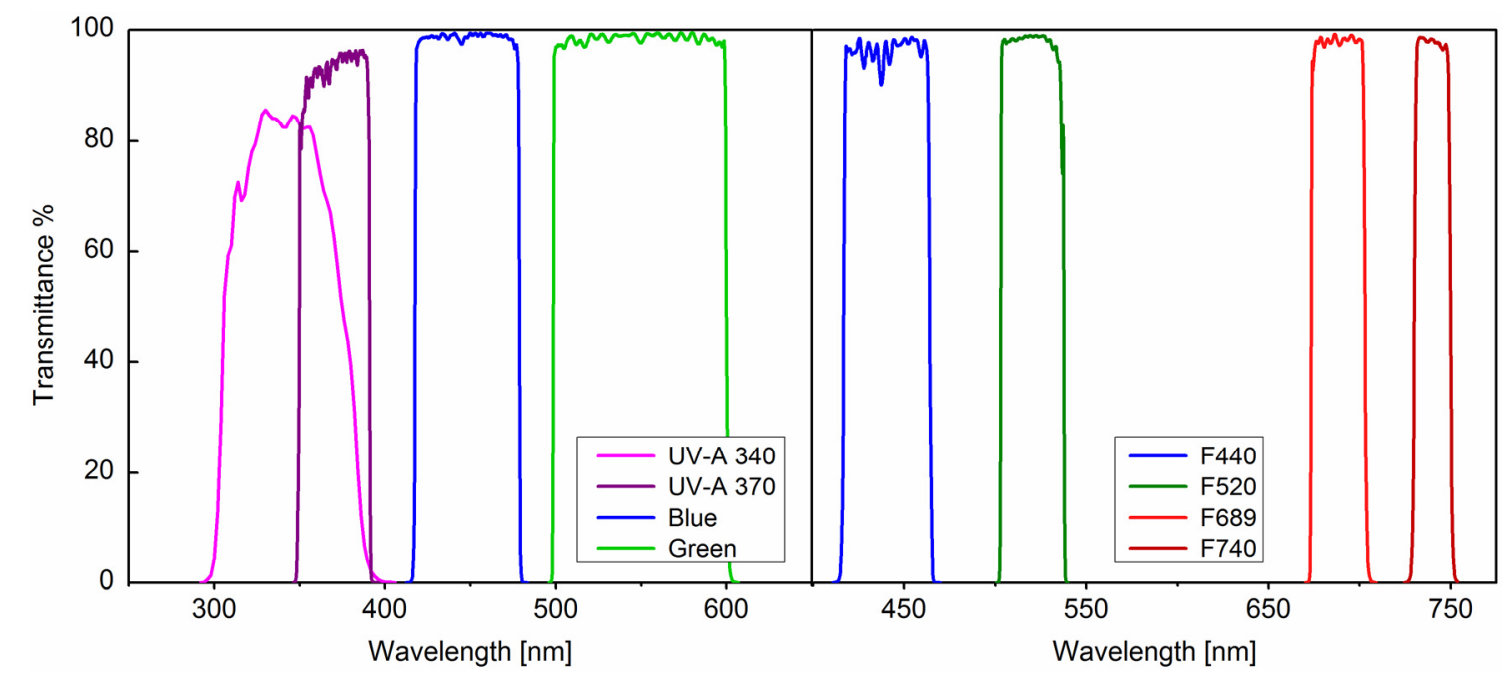




\subsubsection{Detection}

The camera is mounted $0.3 \mathrm{~m}$ horizontally above the sample stage, see Figure 5. Images are recorded using an ICCD camera (C10054-15, Hamamatsu Photonics, Hamamatsu, Japan) with high speed shutter function. It incorporates an image intensifier tube (GaAs photocathode; 1 MCP; P-43 phosphor screen). A CCD video camera module (XC-ES50CE, Sony, Minato, Japan) senses the enhanced signals from the intensifier and has an applicable wavelength range from 370 to $920 \mathrm{~nm}$. Fluorescence images $(768 \times 576$ pixels $)$ are taken from a recording area of $225 \times 169 \mathrm{~mm}\left(38,025 \mathrm{~mm}^{2}\right)$, resulting in a resolution of 7.5 ppi. Directly mounted to the ICCD camera is a lens (Schneider KMP Cinegon, Stemmer Imaging, Puchheim, Germany) with a focal length of $16 \mathrm{~mm}$ (Figure 5). A second filter wheel (AI-FW50-9R, Apogee Imaging System, Roseville, CA, USA), with 9 positions for $50 \mathrm{~mm}$ round filters, is located under the camera-lens assembling (Figure 5). Into this filter wheel, four single-band bandpass filters (BrightLine HC, Semrock, Rochester, NY, USA) are installed with transmittance maxima (Figure 6) in the blue (CWL $440 \mathrm{~nm} / \mathrm{BW} 40 \mathrm{~nm}$ ), green (CWL $520 \mathrm{~nm} /$ BW $28 \mathrm{~nm}$ ), red (CWL 689 nm/BW 23 nm), and near infra-red (CWL 740 nm/BW $13 \mathrm{~nm}$ ).

\subsubsection{Synchronization}

The fine adjustment of the shutter signal of the image intensifier to the real light pulse was carried out using a pulse delay generator (C10149, Hamamatsu Photonics, Hamamatsu, Japan) and the associated software. It gets as input the reference signal from the ignition unit and produces the shutter signal for the image intensifier with adjustable delay (10 ns to $20 \mathrm{~s}$ ) and width ( $5 \mathrm{~ns}$ to $20 \mathrm{~s}$ ). Therefore, it was possible to adjust the rising edge of the shutter signal relative to those of the light pulse better than 50 ns. An optimal signal-to-noise ratio was achieved with an opening width of $7.1 \mu \mathrm{s}$, which is approximately the really width of the light pulse. In this way, the xenon flashes are synchronized with the image intensifier and with the integrating time-sector of the CCD unit of the camera.

\subsubsection{Image Acquisition and Analysis}

The images are recorded with an 8-bit resolution (grayscale), resulting in 7.5 ppi. Image data $(768 \times 576$ pixel) acquired by the camera are transferred to a PC via a frame grabber (Meteor II, Matrox Imaging, Dorval, QC, Canada), where the video data (CCIR format) are digitized and stored. Each image is composed of two subsequent half images (frames), which are read leaving one detector row out. Finally, a complete image consists of the even rows of the first frame and the uneven rows of the second frame. The image acquisition is controlled by special software (BHR Camille, EHR, Pforzheim, Germany), developed for this system. For each sample, 250 and 50 images were accumulated for UV-A excitation and blue/green-excitation, respectively. In all cases, the maximum voltage gain of 900 was used. Afterwards to the image acquisition with excitation light, the same number of images was taken without excitation light (dark signal) and subtracted as background.

Before starting the measurements, a uniformity correction was carried out to eliminate the inhomogeneous illumination by the xenon lamp. For this purpose, an image of the UV-A (370 $\mathrm{nm})$ excited bright blue fluorescence at $440 \mathrm{~nm}$ of white sheet of paper was measured. The software corrects after the plants' fluorescence measurements by using following algorithm (Equation 1): 


$$
C^{*}(x, y)=\frac{C(x, y)}{U(x, y)} \operatorname{mean}(U)
$$

$\mathrm{C}(x, y)$ is the intensity at the coordinates $x$ and $y$ of the current image; $\mathrm{U}(x, y)$ is the intensity at the coordinates $\mathrm{x}$ and $\mathrm{y}$ of the uniformity image; mean $(\mathrm{U})$ is the mean intensity of the uniformity image. $\mathrm{C}^{*}(x, y)$ is the intensity at the coordinates $x$ and $y$ of the resulting image. For this mathematical operation, the images were finally accumulated in one 16-bit resolution image.

For image analysis, the open-source imaging software ImageJ 1.47 [42] was used. The following four shape descriptors were chosen to characterize fungal symptoms using the ImageJ measure-command: Circularity (Equation 2) includes the fraction between area and perimeter. It measures the closeness to a circle, the value of 1 indicating a perfect circle. This parameter is sensitive to changes in overall elongation and boundary roughness.

$$
\text { Circularity }=4 \pi \times \frac{[\text { Area }]}{[\text { Perimeter }]^{2}}
$$

In addition, solidity (Equation 3 ) is sensitive to boundary irregularity, but independent from the size. It is a measurement of the ratio of area to the convex area of the selected region. The convex area is calculated from a convex hull determined by the gift wrap algorithm. The convex hull can be thought of as a rubber band wrapped tightly around the points that define the selection. A high compactness shows values up to 1 .

$$
\text { Solidity }=\frac{[\text { Area }]}{[\text { Convexarea }]}
$$

The aspect ratio (Equation 4) displays the length-width relationship and varies with the overall elongation, but not with outline roughness. Roundness (Equation 5) depends on the area and major axis of the selected area and is so also highly sensitive to elongation. The last two parameters give low values for irregular shapes and higher values for smooth ones.

$$
\begin{gathered}
\text { Aspect ratio }=\frac{[\text { Major axis }]}{[\text { Minor axis }]} \\
\text { Roundness }=4 \times \frac{[\text { Area }]}{\pi \times[\text { Major axis }]^{2}}
\end{gathered}
$$

The resulting fluorescence values were multiplied with individual correction factors each. The following factors were taken into account: (a) relative irradiance of the xenon lamp; (b) spectral transmittance curves of the filters; and (c) sensitivity data of the ICCD camera. Finally, with the chosen filters it is possible to detect four different fluorescence bands (blue, green, red, and far-red fluorescence) using UV light. For example, the UV-A (340 nm) excited blue fluorescence (F440) is abbreviated as ${ }^{340} \mathrm{~F} 440$ in the following text. Blue or green excitation is resulting in two fluorescence bands each: ${ }^{447}$ F689, ${ }^{447}$ F740 and ${ }^{550}$ F689, ${ }^{550}$ F740, respectively. Depending on the experiment two fluorescence bands were used to calculate specific ratios. 


\subsection{Reference Measurements}

The concentration of chlorophylls (Chl $a+b)$ and carotenoids (Car $x+c$ ) was measured by extracting the tissue from the middle part of the leaf (12 $\mathrm{mm}$ long, starting $65 \mathrm{~mm}$ above the leaf base) using $100 \%$ acetone as organic solvent. The sample was homogenized manually and the extract was centrifuged for $300 \mathrm{~s}$ at $2000 \times \mathrm{g}$ in order to exclude turbidity. The resulting supernatant was determined spectrophotometrically using a UV-Vis spectrometer (Specord 210, Analytik Jena, Jena, Germany). The pigment content was calculated using the equations of Lichtenthaler [20].

The Chl content was also characterized in a non-destructive way using a chlorophyll meter (SPAD-502, Minolta, Osaka, Japan). The measurements were carried out at the same site as the pigment determination.

The content of total nitrogen $(\mathrm{N})$ was determined according to the Dumas combustion method using a TruSpec N analyser (LECO Instrumente, Mönchengladbach, Germany). The samples are based on an average of 10 plants for each nitrogen treatment.

\section{Conclusions}

The design of the presented Multi-color imaging system is variable in the distances, and freely accessible filter wheels at the excitation and detection site allow a fast adaptation to new requirements. Additionally, the synchronization between the pulsed xenon flash lamp (excitation source) and camera make it possible to run the system at ambient light conditions, e.g., in the field. If field measurements under full sunlight can be carried out will be a matter of further investigations. Meanwhile the system has been transferred to a partner within the continuing research project CROP.SENSe.net (IGB-2, Forschungszentrum Jülich, Germany) who wants to implement our instrumentation as a part of a multi-sensor system for the greenhouse and on a longer-term version for outdoor measurements.

Excellent fluorescence images can be recorded with the state of the art in excitation light source, extremely sensitive camera, electronic synchronization (between light flashes and camera intensifier in the nanosecond range), and high quality filters (transmittance in restricted spectral ranges close to $100 \%$ ).

Our results demonstrate that an advanced multi-color imaging system, based on chlorophyll fluorescence and blue-green fluorescence, in combination with an extended data analysis (here shape descriptors), is also capable of detecting and characterizing local stress symptoms. This approach has the potential to provide new applications for sensor systems that can be assessed remotely in a precision agriculture context, especially when several stress factors are involved. Further studies, particular in the data analysis, are still needed to establish reliable fluorescence image sensors to answer complex questions for detection and discrimination of biotic and abiotic stresses under field conditions.

\section{Acknowledgments}

This study was performed within the research project CROP.SENSe.net ("Networks of excellence in agricultural and nutrition research") funded by the German Federal Ministry of Education and Research (BMBF 0315532B). The authors would like to thank Mario Müller and Markus Mokry (LTZ Augustenberg, Karlsruhe, Germany) for kindly cultivating the barley plants under different 
nitrogen conditions. We also like to thank Katja Herzog and Reinhard Töpfer (JKI Siebeldingen, Germany) for providing the inoculated grapevine leaf samples.

\section{Author Contributions}

Stefanie Konanz has carried out assembling, testing and measurements with the Fluorescence Imaging System. She also performed the data analysis. László Kocsányi has conceived the configuration of the components of the system and in particular in the development of the light source and its synchronisation with the camera. Claus Buschmann established the general concept of the fluorescence measurement and helped with data interpretation. All authors read and approved the final manuscript.

\section{Conflicts of Interest}

The authors declare no conflict of interest.

\section{References}

1. Valeur, B.; Berberan-Santos, M.N. Molecular Fluorescence-Principles and Applications, 2nd ed.; Wiley-VCH: Weinheim, Germany, 2012; pp. 1-569.

2. Rost, F.W.D. Autofluorescence in Plants, Fungi and Bacteria. In Fluorescence Microscopy; Cambridge University Press: Cambridge, UK, 1995; Volume 2, pp. 16-39.

3. Morales, F.; Cerovic, Z.G.; Moya, I. Time-resolved blue-green fluorescence of sugar beet (Beta vulgaris L.) leaves. Spectroscopic evidence for the presence of ferulic acid as the main fluorophore of the epidermis. Biochim. Biophys. Acta 1996, 1273, 251-262.

4. Lichtenthaler, H.K.; Schweiger, J. Cell wall bound ferulic acid, the major substance of the blue-green fluorescence emission of plants. J. Plant Physiol. 1998, 152, 272-282.

5. Nicholson, R.L.; Hammerschmidt, R. Phenolic compounds and their role in disease resistance. Annu. Rev. Phytopathol. 1992, 30, 369-389.

6. Dixon, R.A.; Paiva, N.L. Stress-induced phenylpropanoid metabolism. Plant Cell 1995, 7, 1085-1097.

7. Treutter, D. Significance of flavonoids in plant resistance: A review. Environ. Chem. Lett. 2006, 4, 147-157.

8. Bilger, W.; Veit, M.; Schreiber, L.; Schreiber, U. Measurement of leaf epidermal transmittance of UV radiation by chlorophyll fluorescence. Physiol. Plant 1997, 101, 754-763.

9. Buschmann, C. Variability and application of the chlorophyll fluorescence emission ratio red/far-red of leaves. Photosynth. Res. 2007, 92, 261-271.

10. Papageorgiou, G.C.; Govindjee. Chlorophyll a Fluorescence: A Signature of Photosynthesis; Springer: Dordrecht, The Netherlands, 2004; pp. 1-818.

11. Omasa, K.; Shimazaki, K.-I.; Aiga, I.; Larcher, W.; Onoe, M. Image analysis of chlorophyll fluorescence transients for diagnosing the photosynthetic system of attached leaves. Plant Physiol. 1987, 84, 748-752. 
12. Buschmann, C.; Lichtenthaler, H.K. Principles and characteristics of multi-colour fluorescence imaging of plants. J. Plant Physiol. 1998, 152, 297-314.

13. Nedbal, L.; Whitmarsh, J. Chlorophyll Fluorescence Imaging of Leaves and Fruits. In Chlorophyll a Fluorescence: A Signature of Photosynthesis; Papageorgiou, G.C., Govindjee, Eds.; Springer: Dordrecht, The Netherlands, 2004; pp. 389-407.

14. Buschmann, C.; Langsdorf, G.; Lichtenthaler, H.K. Blue, Green, Red and Far-red Fluorescence Signatures of Plant Tissues, Their Multicolor Fluorescence Imaging, and Application for Agrofood Assessment. In Optical Monitoring of Fresh and Processed Agricultural Crops; Zude, M., Ed.; CRC-Press Taylor \& Francis Group: Boca Raton, FL, USA, 2008; pp. 272-319.

15. Chaerle, L.; Lenk, S.; Leinonen, I.; Jones, H.G.; van Der Straeten, D.; Buschmann, C. Multi-sensor plant imaging: Towards the development of a stress-catalogue. Biotechnol. J. 2009, 4, 1152-1167.

16. Lenk, S.; Buschmann, C. Distribution of UV-shielding of the epidermis of sun and shade leaves of the beech (Fagus sylvatica L.) as monitored by multi-colour fluorescence imaging. J. Plant Physiol. 2006, 163, 1273-1283.

17. Schneider, C.A.; Rasband, W.S.; Eliceiri, K.W. NIH Image to ImageJ: 25 Years of image analysis. Nat. Methods 2012, 9, 671-675.

18. Konanz, S. Botanical Institute II, Karlsruhe Institute of Technology (KIT), Karlsruhe, Germany. Unpublished work, 2012.

19. Langcake, P.; Pryce, R.J. The production of resveratrol by Vitis vinifera and other members of the Vitaceae as a response to infection or injury. Physiol. Plant Pathol. 1976, 9, 77-86.

20. Lichtenthaler, H.K. Chlorophylls and carotenoids: Pigments of photosynthetic biomembranes. Methods Enzymol. 1987, 148, 350-382.

21. Hák, R.; Rinderle-Zimmer, U.; Lichtenthaler, H.K.; Nátr, L. Chlorophyll $a$ fluorescence signatures of nitrogen deficient barley leaves. Photosynthetica 1993, 28, 151-159.

22. Khamis, S.; Lamaze, T.; Lemoine, Y.; Foyer, C. Adaptation of the photosynthetic apparatus in maize leaves as a result of nitrogen limitation. Relationships between electron transport and carbon assimilation. Plant Physiol. 1990, 94, 1436-1443.

23. Langsdorf, G.; Buschmann, C.; Sowinska, M.; Babani, F.; Mokry, M.; Timmermann, F.; Lichtenthaler, H.K. Multicolour fluorescence imaging of sugar beet leaves with different nitrogen status by flash lamp UV-excitation. Photosynthetica 2000, 38, 539-551.

24. Ciompi, S.; Gentili, E.; Guidi, L.; Soldatini, G.F. The effect of nitrogen deficiency on leaf gas exchange and chlorophyll fluorescence parameters in sunflower. Plant Sci. 1996, 118, 117-184.

25. Shangguan, Z.; Shao, M.; Dyckmans, J. Effects of nitrogen nutrition and water deficit on net photosynthetic rate and chlorophyll fluorescence in winter wheat. J. Plant Physiol. 2000, 156, 46-51.

26. Konanz, S.; Buschmann, C. Botanical Institute II, Karlsruhe Institute of Technology (KIT), Karlsruhe, Germany. Unpublished work, 2012.

27. Takahashi, K.; Mineuchi, K.; Nakamura, T.; Koizumi, M.; Kano, H. A system for imaging transverse distribution of scattered light and chlorophyll fluorescence in intact rice leaves. Plant Cell Environ. 1994, 17, 105-110. 
28. Koizumi, M.; Takahashi, K.; Mineuchi, K.; Nakamura, T.; Kano, H. Light gradients and the transverse distribution of chlorophyll fluorescence in mangrove and Camellia leaves. Ann. Bot. 1998, 81, 527-533.

29. Terashima, I.; Fujita, T.; Inoue, T.; Chow, W.S.; Oguchi, R. Green light drives leaf photosynthesis more efficiently than red light in strong white light: Revisiting the enigmatic question of why leaves are green. Plant Cell Physiol. 2009, 50, 684-679.

30. Brodersen, C.R.; Vogelmann, T.C. Do changes in light direction affect absorption profiles in leaves? Funct. Plant Biol. 2010, 37, 403-412.

31. Lichtenthaler, H.K.; Rinderle, U. The role of chlorophyll fluorescence in the detection of stress conditions in plants. Crit. Rev. Anal. Chem. 1988, 19, 29-85.

32. Govindjee; van de Ven, M.; Cao, J.; Roye, C.; Gratton, E. Multifrequency cross-correlation phase fluorometry of chlorophyll $a$ fluorescence in thylakoid and PSII enriched membranes. Photochem. Photobiol. 1993, 58, 438-445.

33. Gitelson, A.A.; Buschmann, C.; Lichtenthaler, H.K. Leaf chlorophyll fluorescence corrected for re-absorption by means of absorption and reflectance measurements. J. Plant Physiol. 1998, 152, 283-296.

34. Hák, R.; Lichtenthaler, H.K.; Rinderle, U. Decrease of the chlorophyll fluorescence ratio F690/F730 during greening and development of leaves. Radiat. Environ. Biophys. 1990, 29, 329-336.

35. Lichtenthaler, H.K.; Hák, R.; Rinderle, U. The chlorophyll fluorescence ratio F690/F730 in leaves of different chlorophyll content. Photosynth. Res. 1990, 25, 295-298.

36. D’Ambrosio, N.; Szabo, K.; Lichtenthaler, H.K. Increase of the chlorophyll fluorescence ratio F690/F735 during the autumnal chlorophyll breakdown. Radiat. Environ. Biophys. 1992, 31, 51-62.

37. Babani, F.; Lichtenthaler, H.K. Light-induced and age-dependent development of chloroplasts in etiolated barley leaves as visualized by determination of photosynthetic pigments, $\mathrm{CO}_{2}$ assimilation rates and different kinds of chlorophyll fluorescence ratios. J. Plant Physiol. 1996, $148,555-566$.

38. Agati, G. Response of the in vivo chlorophyll fluorescence spectrum to environmental factors and laser excitation wavelength. Pure Appl. Opt. 1998, 7, 797-807.

39. Cerovic, Z.G.; Ounis, A.; Cartelat, A.; Latouche, G.; Goulas, Y.; Meyer, S.; Moya, I. The use of chlorophyll fluorescence excitation spectra for the non-destructive in situ assessment of UV-absorbing compounds in leaves. Plant Cell Environ. 2002, 25, 1663-1676.

40. Buschmann, C.; Langsdorf, G.; Lichtenthaler, H.K. Imaging of the blue, green, and red fluorescence emission of plants: An overview. Photosynthetica 2000, 38, 483-491.

41. Bilger, W.; Johnson, T.; Schreiber, U. UV-excited chlorophyll fluorescence as a tool for the assessment of UV-protection by the epidermis of plants. J. Exp. Bot. 2001, 52, 2007-2014.

42. Rasband, W.S. ImageJ; US National Institutes of Health: Bethesda, MD, USA, 1997-2012; Available online: http://imagej.nih.gov/ij/ (accessed on 11 March 2013).

(C) 2014 by the authors; licensee MDPI, Basel, Switzerland. This article is an open access article distributed under the terms and conditions of the Creative Commons Attribution license (http://creativecommons.org/licenses/by/3.0/). 